\title{
Fractionally Spread Multicarrier CDMA: A Multiple-Access Scheme for Various Wireless Channels
}

\author{
Lie-Liang Yang and Lajos Hanzo \\ Dept. of ECS, University of Southampton, SO17 1BJ, UK. \\ Tel: +44-23-8059 3125, Fax: +44-23-8059 4508 \\ Email: lly,lh@ecs.soton.ac.uk; http://www-mobile.ecs.soton.ac.uk
}

\begin{abstract}
In multicarrier code-division multiple-access (MCCDMA) the total system bandwidth is divided into a number of subbands, where each subband may use direct-sequence (DS) spreading and each subband signal is transmitted using a subcarrier frequency. In this contribution we divide the symbol duration into a number of fractional sub-symbol durations also referred to here as fractions, in a manner analogous to subbands in MCCDMA systems. In the proposed MC-CDMA scheme the data streams are spread at both the symbol-fraction level and at the chip level by the transmitter, and hence the proposed scheme is referred to as the fractionally-spread MC-CDMA arrangement, or FS MC-CDMA. In comparison to conventional MC-CDMA schemes, which are suitable for communications over frequencyselective fading channels, our study demonstrates that the proposed FS MC-CDMA is capable of efficiently exploiting both the frequency-selective and the time-selective characteristics of wireless channels.
\end{abstract}

\section{INTRODUCTION}

In MC-CDMA communication systems [1]-[7], the total bandwidth available is divided into a number of subbands, where each subband may employ direct-sequence (DS) spreading and each subband signal is transmitted with the aid of a subcarrier. In MC-CDMA systems [3], [5], [6] serial-to-parallel (S-P) conversion is invoked at the transmitter, in order to decrease the transmitted symbol rate for the sake of mitigating the effects of inter-symbol interference (ISI). Frequency diversity in MC-CDMA systems is usually achieved by repeating the transmitted signal in the frequency (F)-domain with the aid of several subcarriers [4], [5], [7]. Classic studies of MC-CDMA have shown that the various MC-CDMA schemes proposed in the literature [2]-[7] are capable of supporting multiple users communicating over frequency-selective fading channels.

However, the future generations of broadband multipleaccess communication systems [8] are expected to have a bandwidth on the order of tens or even hundreds of MHz. Broadband wireless mobile channels are typically time-varying and the received signals may experience both frequency-selective and time-selective fading [9], [10]. Since conventional MCCDMA schemes have usually been designed without consid-

This work has been funded in the framework of the IST project IST-200134091 SCOUT, which is partly funded by the European Union. The authors would like to acknowledge the contributions of their colleagues. ering the time-selectivity of the wireless channels, they may not be sufficiently efficient, when communicating over wireless channels exhibiting both frequency-selective and time-selective fading. In this contribution, firstly, we resort to multicarrier transmission of the DS-CDMA signals for the sake of achieving frequency diversity, as considered in [4], [5]. Secondly, in a manner analogous to the approach used in MC DS-CDMA schemes [4]- which divide the available bandwidth into a number of subbands - we divide the symbol-duration into a number of sub-symbol-durations referred to here as fractions, for the sake of achieving time diversity, when communicating over fast-fading channels. Hence, the proposed scheme is referred to as fractionally spread MC-CDMA ( FS MC-CDMA).

Our study and results demonstrate that the proposed FS MCCDMA is capable of efficiently exploiting both the frequencyselective and the time-selective characteristics of the wireless channels encountered for enhancing the achievable BER performance. Furthermore, the proposed FS MC-CDMA has the potential of flexibly achieving the best balance between the attainable spreading gain and diversity gain. The diversity gain over frequency-selective fast fading channels automatically converts to the spreading gain without requiring external reconfiguration, when the frequency-selectivity and/or time-selectivity of wireless channels becomes less dominant. Owing to the above self-flexible properties, the proposed FS MC-CDMA scheme is beneficial for communications over wireless channels exhibiting frequency-selective fading and/or time-selective fading.

\section{System ModeL}

\section{A. Transmitted Signals}

The transmitter diagram of the $k$ th user is shown in Fig.1 for the proposed FS MC-CDMA system. In this scheme the original binary data stream having a bit duration of $T_{b}$ is SP converted to $U$ parallel substreams, which are expressed as $\left\{b_{k 1}, b_{k 2}, \ldots, b_{k U}\right\}$. The new bit duration or symbol duration after $\mathrm{S}-\mathrm{P}$ conversion is given by $T_{s}=U T_{b}$. As shown in Fig.1, after S-P conversion each of the substreams is spread using two time (T)-domain spreading codes, namely $a_{k}(t)$ and $c_{k}(t)$. More explicitly, the first T-domain spreading code $a_{k}(t)$ is applied at the fraction level and it is expressed as $a_{k}(t)=$ 


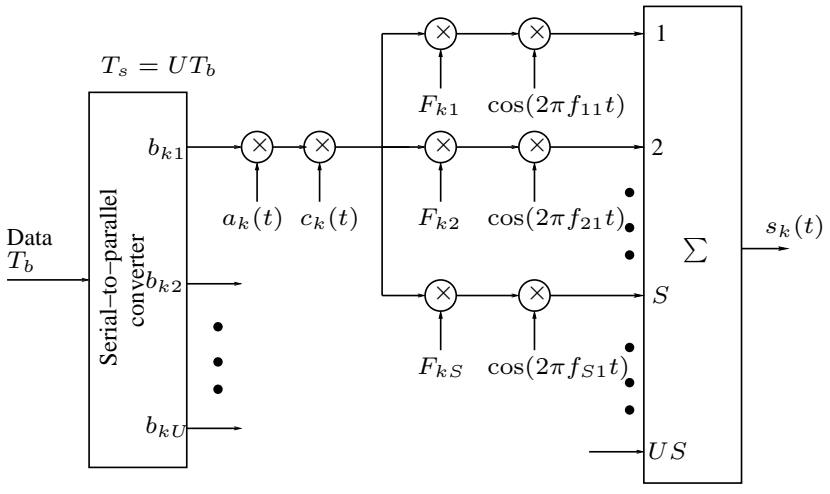

Fig. 1. Transmitter model of fractionally spread MC-CDMA.

$\sum_{i=-\infty}^{\infty} a_{k i} P_{T_{D}}\left(t-i T_{D}\right)$, where $T_{D}=T_{s} / N_{1}$ represents the fraction's time-duration, $a_{k i}$ assumes the binary values of +1 or -1 with equal probability, while $P_{T_{D}}(t)$ represents the rectangular pulses of duration $T_{D}$. Hence, $a_{k}(t)$ consists of a sequence of mutually independent rectangular pulses of duration $T_{D}$ and amplitude of +1 or -1 , both having an equal probability. The second T-domain spreading code $c_{k}(t)$ is at the chip level and is defined as $c_{k}(t)=\sum_{i=-\infty}^{\infty} c_{k i} P_{T_{c}}\left(t-i T_{c}\right)$, where $\left\{c_{k i}\right\}$ is again a random sequence with $c_{k i} \in\{+1,-1\}$ and $P_{T_{c}}(t)$ is the rectangular chip waveform defined over the time interval $\left[0, T_{c}\right)$.

Let the total number of subcarrier frequencies, namely $U S$, where $S$ is defined as the length of the F-domain spreading codes to be invoked additionally. We assume that the spacing between two adjacent subcarrier frequencies is $2 / T_{c}$ and there exists no overlap among the main spectral lobes of the subcarriers. As shown in Fig.1, after T-domain spreading the $u$ th substream, where $u=1,2, \ldots, U$, is further spread in the frequency (F)-domain using an $S$-chip F-domain spreading code $\left\{F_{k 1}, F_{k 2}, \ldots, F_{k S}\right\}$ associated with the $S$ number of subcarrier frequencies of $\left\{f_{u 1}, f_{u 2}, \ldots, f_{u S}\right\}$. Finally, the $U S$ number of subcarrier-modulated substreams are superimposed on each other in order to form the transmitted signal, which can be expressed as

$$
\begin{array}{r}
s_{k}(t)=\sqrt{\frac{2 E_{b}}{T_{s} S}} \sum_{u=1}^{U} \sum_{s=1}^{S} b_{k u}(t) a_{k}(t) c_{k}(t) F_{k s} \\
\times \cos \left(2 \pi f_{u s} t+\phi_{u s}^{(k)}\right),
\end{array}
$$

where $E_{b}$ represents the energy per bit, $b_{k u}(t)$ denotes the $u$ th binary data's waveform after the S-P conversion, while $\phi_{u s}^{(k)}$ represents a random phase due to carrier modulation. Assuming $N_{2}=T_{D} / T_{c}$ being an integer, then the total T-domain spreading factor is $N=T_{s} / T_{c}=T_{s} / T_{D} \times T_{D} / T_{c}=N_{1} N_{2}$.

\section{RECEIVER MODEL}

we assume that the delay-spread of the channel, denoted by $T_{m}$ is lower than the chip-duration $T_{c}$, i.e., we have $T_{m}<T_{c}$. In practise the condition of $T_{m}<T_{c}$ can be achieved by employing high chip-duration spreading sequences for each of the subcarriers, but assigning an increased number of subcarriers. The required frequency diversity again is achieved by transmitting the same data on several subcarriers experiencing independent fading. Since we assume that $T_{m}<T_{c}$, the number of resolvable paths associated with each subcarrier is therefore one, i.e., each subcarrier signal experiences flat fading. As shown in Fig.1, the FS MC-CDMA transmitter usually employs S-P conversion and $U$ data bits are transmitted in parallel within each symbol-duration. Hence, the symbol-duration is $T_{s}=U T_{b}$. For rapidly time-varying wireless channels, which may be encountered by high-velocity mobile terminals or fast moving large-bodied objects in the vicinity of the mobile terminal, the fading amplitude may change significantly within a given symbol-duration, resulting in high-Doppler timeselective fading. Therefore, for fast fading channels we assume that the fading amplitude within each fraction of $T_{D}$ is a constant, while the received signal experiences independent fading during each fraction. However, if the fractions are subject to correlated fading, then interleaving over time can be employed before the S-P conversion stage of Fig.1, in order to guarantee the independent fading of the subcarrier signals in each fraction. Based on the above assumptions, the asynchronous signal received by the base station can be expressed as

$$
\begin{array}{r}
r(t)=\sqrt{\frac{2 E_{b}}{T_{s} S}} \sum_{k=1}^{K} \sum_{n=-\infty}^{\infty} \sum_{u=1}^{U} \sum_{s=1}^{S} \alpha_{u n s}^{(k)} b_{k u}\left(t-\tau_{k}\right) a_{k n} \\
\times P_{T_{D}}\left(t-n T_{D}-\tau_{k}\right) \times c_{k}\left(t-\tau_{k}\right) F_{k s} \\
\times \cos \left(2 \pi f_{u s} t+\psi_{\text {uns }}^{(k)}\right)+n(t),
\end{array}
$$

where $n(t)$ represents the AWGN noise having zero mean and a double-sided power spectrum density of $\mathcal{N}_{0} / 2$. Furthermore, in (2) $\alpha_{u n s}^{(k)}$ is an amplitude fading parameter associated with the $k$ th user, with the $n$th fraction as well as with the subcarrier indexed by the values of $u$ and $s$. Note that, $\alpha_{u n s}^{(k)}=1$, when nonfading AWGN channels are considered, while $\alpha_{u n s}^{(k)}=\alpha_{u s}^{(k)}$, i.e. it is independent of the fraction index of $n$, when considering frequency-selective slow fading channels.

Let the first user be the user-of-interest and let us ignore the subscript as well as the superscript associated with the reference user. The transmitter of Fig.1 and (1) transmits each data bit on $S$ number of subcarriers using $N_{1}$ fractions. At the receiver side, these $N_{1} S$ number of signals conveying the same data bit are combined based on the maximum ratio combining (MRC) principle, when assuming rapidly fading channels. Therefore, we have to estimate both the fading amplitude $\alpha_{u n s}$ and the phase $\psi_{u n s}$ associated with each of the subcarrier signals within each fraction. In this contribution we assume 


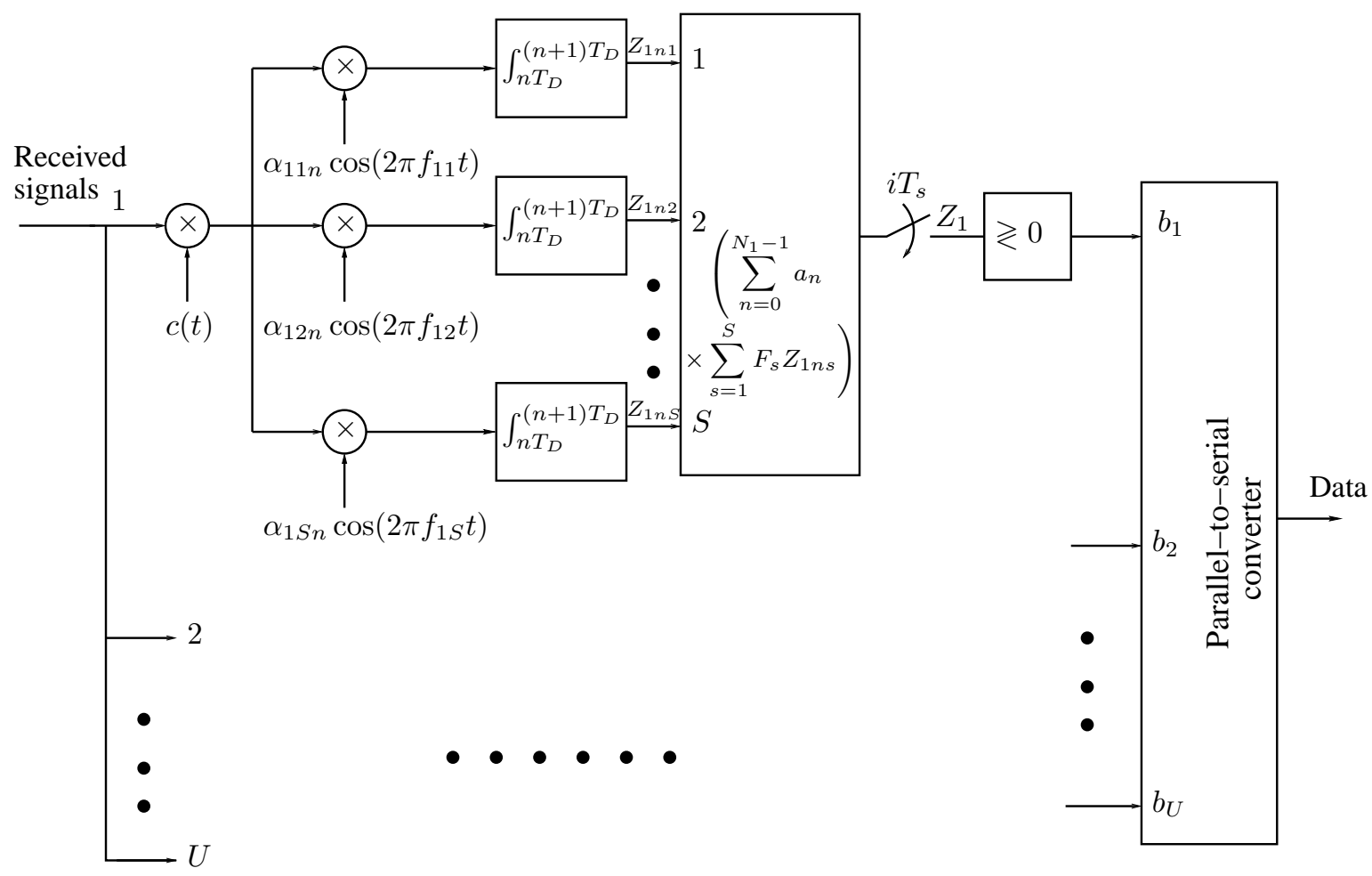

Fig. 2. Receiver schematic diagram of fractionally spread MC-CDMA.

that these channel parameters are perfectly estimated. Note that when AWGN channels are considered, the fading amplitudes of $\left\{\alpha_{u n s}\right\}$ are set to units, while the phases due to carrier modulation and channel delay are assumed to be perfect estimates. By contrast, for the slowly fading channels considered, both the fading amplitudes as well as the phases are independent of the fraction index of $n$. The FS MC-CDMA receiver's schematic diagram is shown in Fig.2, which is suitable for receiving the FS MC-CDMA signals in all three types of channel models considered. In Fig.2 each subcarrier signal is first despread using the T-domain spreading code $c(t)$ of the reference user associated with each fraction. Then, the subcarrier signals conveying the same data bit are despread using the F-domain spreading code $\left\{F_{1}, F_{2}, \ldots, F_{S}\right\}$ and combined using a MRC scheme with the aid of the channel's fading envelope estimates $\left\{\alpha_{u n 1}, \alpha_{u n 2}, \ldots, \alpha_{u n S}\right\}_{u=1}^{U}$. Finally, the $N_{1}$ number of signals corresponding to $N_{1}$ fractions of the same symbol are despread using the T-domain spreading code $a(t)$, yielding the decision variable $Z_{u}, u=1, \ldots, U$ acquired for the $u$ th binary bit. The process of generating the decision variable $Z_{u}$ for the first symbol can be summarized using the following equations.

$$
\begin{aligned}
Z_{u} & =\sum_{n=0}^{N_{1}-1} a_{n} Z_{u n}, u=1, \ldots, U, \\
Z_{u n} & =\sum_{s=1}^{S} F_{s} Z_{u n s},
\end{aligned}
$$

$$
Z_{u n s}=\alpha_{u n s} \times \int_{n T_{D}}^{(n+1) T_{D}} r(t) c(t) \cos \left(2 \pi f_{u s} t\right) d t,
$$

where we assumed that $\tau_{1}=0$ and $\psi_{\text {uns }}=0$, representing perfect synchronization with the subcarrier signal of the fraction that is being considered.

\section{BIt ERROR RATE}

In this section we summarize the BER expressions for the proposed FS MC-CDMA system, when communicating over the AWGN, and over the slow or fast frequency-selective Nakagami- $m$ fading channels.

The BER of the FS MC-CDMA system communicating over AWGN channels can be expressed as

$$
\begin{aligned}
P_{b} & =Q(\sqrt{2 \cdot \mathrm{SINR}}) \\
& =Q\left(\left[\frac{K-1}{3 N_{1} N_{2} S}+\left(\frac{2 E_{b}}{\mathcal{N}_{0}}\right)^{-1}\right]^{-1 / 2}\right),
\end{aligned}
$$

where $Q(x)$ represents the Gaussian $Q$-function, which can be represented in the form of $Q(x)=\frac{1}{\sqrt{2 \pi}} \int_{x}^{\infty} \exp \left(-\frac{t^{2}}{2}\right) d t$.

In the context of the frequency-selective fast Nakagami- $m$ fading channels, the BER of the FS MC-CDMA systems can be expressed as

$$
P_{b}=\frac{1}{\pi} \int_{0}^{\pi / 2}\left(\frac{m \sin ^{2} \theta}{\gamma_{c}+m \sin ^{2} \theta}\right)^{m S N_{1}} d \theta
$$


where $m$ is the Nakagami- $m$ fading parameter, $\gamma_{c}$ represents the average signal-to-noise ratio (SNR) received and can be expressed as

$$
\gamma_{c}=\left[\frac{2(K-1)}{3 N_{2}}+\left(\frac{\Omega E_{b}}{S N_{1} \mathcal{N}_{0}}\right)^{-1}\right]^{-1},
$$

where $\Omega=E\left[\left(\alpha_{u n s}^{(k)}\right)^{2}\right]$. Explicitly, (7) shows that the diversity order achieved is $S N_{1}$.

Finally, in the context of communicating over frequencyselective slow Nakagami- $m$ fading channels, the BER of the FS MC-CDMA systems can be expressed as

$$
P_{b}=\frac{1}{\pi} \int_{0}^{\pi / 2}\left(\frac{m \sin ^{2} \theta}{\gamma_{c}+m \sin ^{2} \theta}\right)^{m S} d \theta,
$$

which shows the diversity order achieved over the frequencyselective slow Nakagami- $m$ fading channels is $S$. Furthermore, the SNR in (9) is given by

$$
\gamma_{c}=\left[\frac{2(K-1)}{3 N_{1} N_{2}}+\left(\frac{\Omega E_{b}}{S \mathcal{N}_{0}}\right)^{-1}\right]^{-1} .
$$

It can be shown [6] that the limit of (7) or (9) with respect to $m \rightarrow \infty$ will converge to (6), which quantifies the BER in the context of AWGN channels. This characteristic implies that when the channel quality improves and the fading envelope becomes near-constant, the FS MC-CDMA will automatically leverage the diversity gain into spreading gain.

\section{Performance Results and Discussion}

In Fig. 3 we show the corresponding comparison of the BER performance of the FS MC-CDMA system, when communicating over both the non-fading AWGN, as well as over the frequency-selective slow fading and fast fading channels, assuming both Rayleigh $(m=1)$ and Nakagami- $m(m=2)$ fading models. The curves in the figure were plotted against the average SNR per bit of $E_{b} / \mathcal{N}_{0}$ for the parameters of $N_{1}=$ 4, $N_{2}=31, S=4$ and $K=30$. From the results of Fig.3 we observe that for a given SNR per bit value, the frequencyselective fast fading channel model achieves a lower BER, than the frequency-selective slow fading channel model, regardless of $m=1$ or $m=2$. Furthermore, for $m=1$ or $m=2$, the BER performance curve of the frequency-selective fast fading channel model is only about $4 \mathrm{~dB}$ or $2 \mathrm{~dB}$ away from the BER performance curve of the AWGN channel, at the BER of $10^{-6}$, respectively. By contrast, at the same BER of $10^{-6}$, the BER performance of the frequency-selective slow fading channel is significantly worse than that over AWGN channels, regardless of $m=1$ and $m=2$. For $m=1$ or $m=2$ we observe the formation of an error floor for the frequency-selective slow fading channel model at the SNR per bit values of about $20 \mathrm{~dB}$

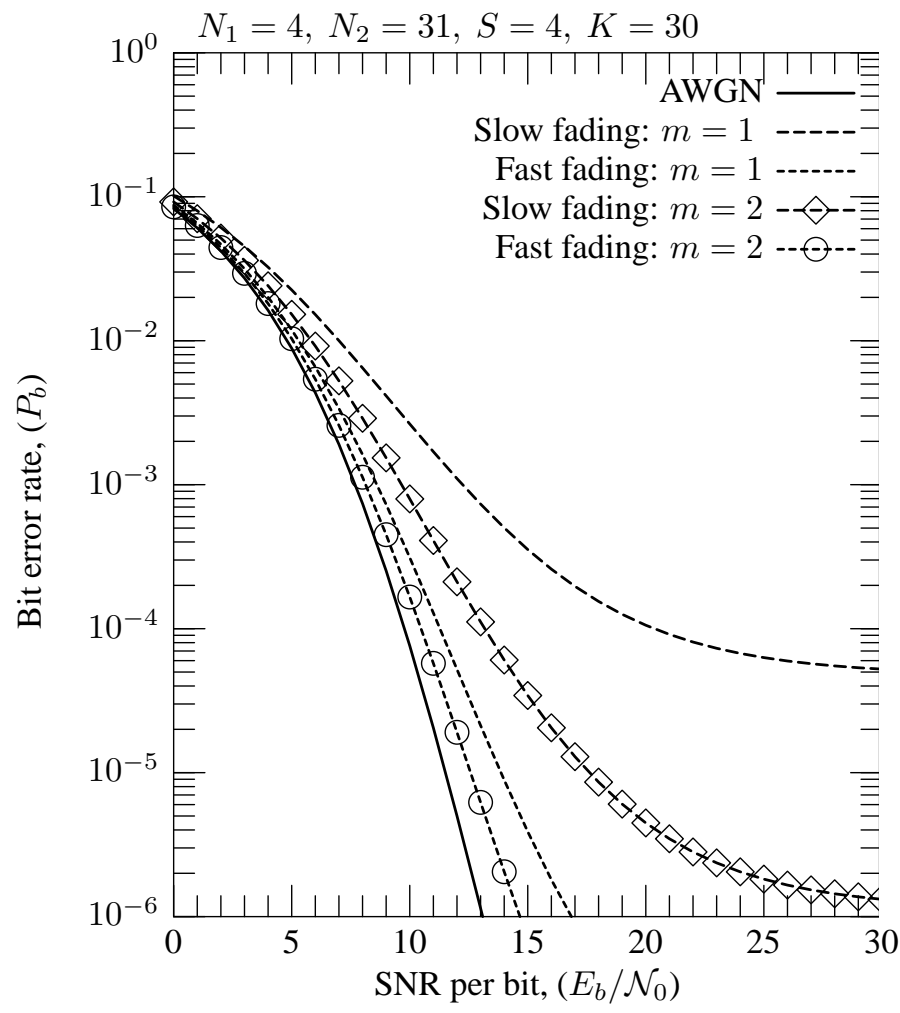

Fig. 3. BER versus $E_{b} / \mathcal{N}_{0}$ performance of the FS-MC-CDMA systems, when communicating over both non-fading AWGN, as well as over slow frequencyselective and fast frequency-selective Rayleigh $(m=1)$ or Nakagami- $m(m=$ 2) fading channels.

or $25 \mathrm{~dB}$, respectively. The reason for the performance trends of Fig. 3 is that in the context of the frequency-selective fast fading channel model the total diversity order is $S N_{1}=16$. By contrast, in the context of the frequency-selective slow fading channel model, the diversity order is only $S=4$.

Fig.4 demonstrates the comparison of the BER performance versus the number of users $K$ for the FS MC-CDMA system, when communicating over both non-fading AWGN, as well as over frequency-selective slow Rayleigh $(m=1)$ fading and fast Rayleigh $(m=1)$ fading channels. The curves in Fig.4 were plotted versus the number of users $K$ for the parameters of $N_{1}=4, N_{2}=31, S=4$ and $E_{b} / \mathcal{N}_{0}=15 \mathrm{~dB}$. The results of Fig. 4 also show that the achievable BER performance is better in frequency-selective fast fading environments, than in the frequency-selective slow fading environments. This is a consequence of the higher diversity order achievable over the frequency-selective fast fading channels, than over the frequency-selective slow fading channels.

Finally, Fig.5 shows the BER performance of the FS MCCDMA system with respect to the Nakagami fading parameter $m$, when communicating over frequency-selective slow or fast Nakagami- $m$ fading channels. For the sake of comparison, the benchmark BER performance achieved in AWGN channels was also plotted in Fig.5. The results of Fig.5 illustrate that when the value of $m$ increases, the BER performance of FS MC-CDMA 


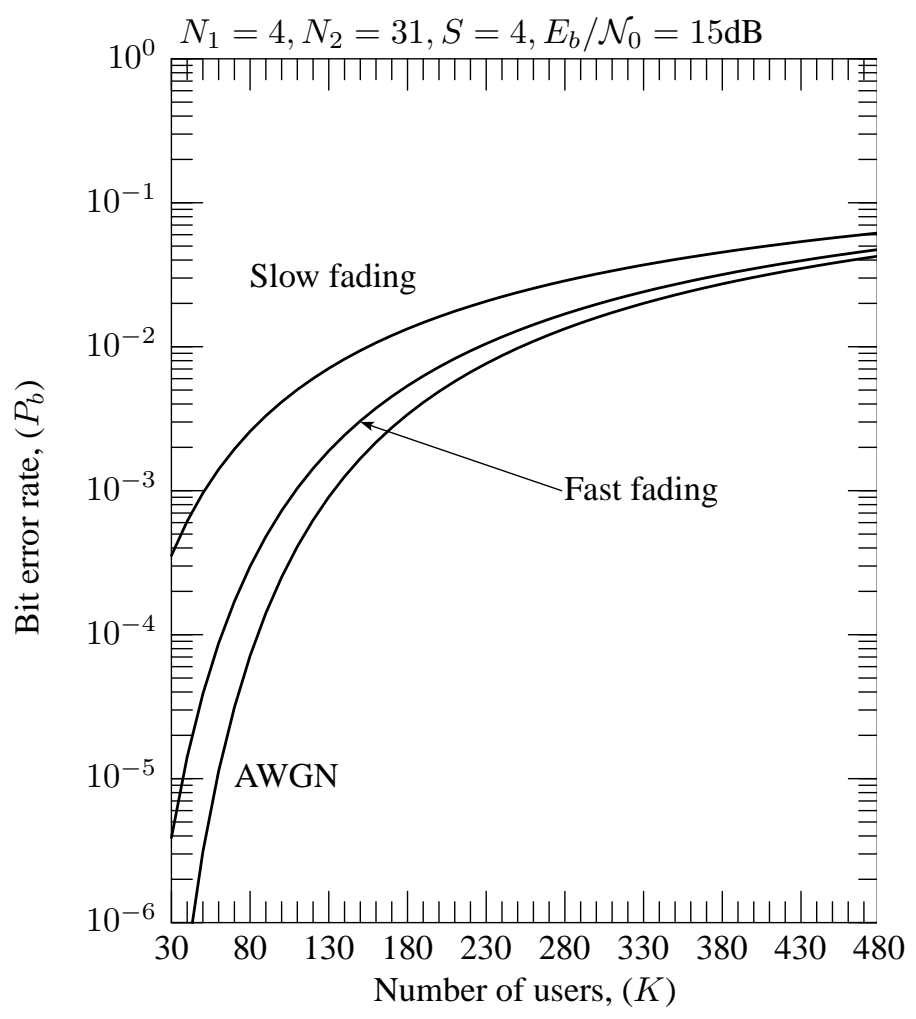

Fig. 4. BER performance versus the number of users $K$ for the FS-MCCDMA systems, when communicating over both non-fading AWGN, as well as over slow frequency-selective and fast frequency-selective Rayleigh $(m=1)$ fading channels.

over both fast and slow fading channels will approach the BER performance of AWGN channels. However, for any given fading parameter $m$, the FS MC-CDMA system communicating over fast fading channels is capable of achieving a lower BER, than over slow fading channels.

In summary, in this contribution we have proposed a novel fractionally spread multicarrier CDMA arrangement, i.e., the FS MC-CDMA scheme, which employs concatenated Tdomain spreading for improving the achievable performance, when communicating over wireless channels exhibiting both frequency-selective and time-selective fading. It can be shown that the proposed FS MC-CDMA constitutes a self-flexible scheme without requiring external reconfiguration. It is capable of automatically converting the spreading gain to the diversity gain, when the frequency-selectivity and/or time-selectivity of the wireless channel becomes more dominant. Conversely, it has the ability to leverage the diversity gain into the spreading gain, when the frequency-selectivity and/or time-selectivity of the wireless channel becomes less prevalent. Furthermore, our numerical results show that in FS MC-CDMA the BER performance attained, when communicating over fast fading channels is significantly better than that over slow fading channels. The BER performance achieved over fading channels approaches that achievable over AWGN channels, when the grade of frequency-selectivity and/or time-selectivity of the fad-

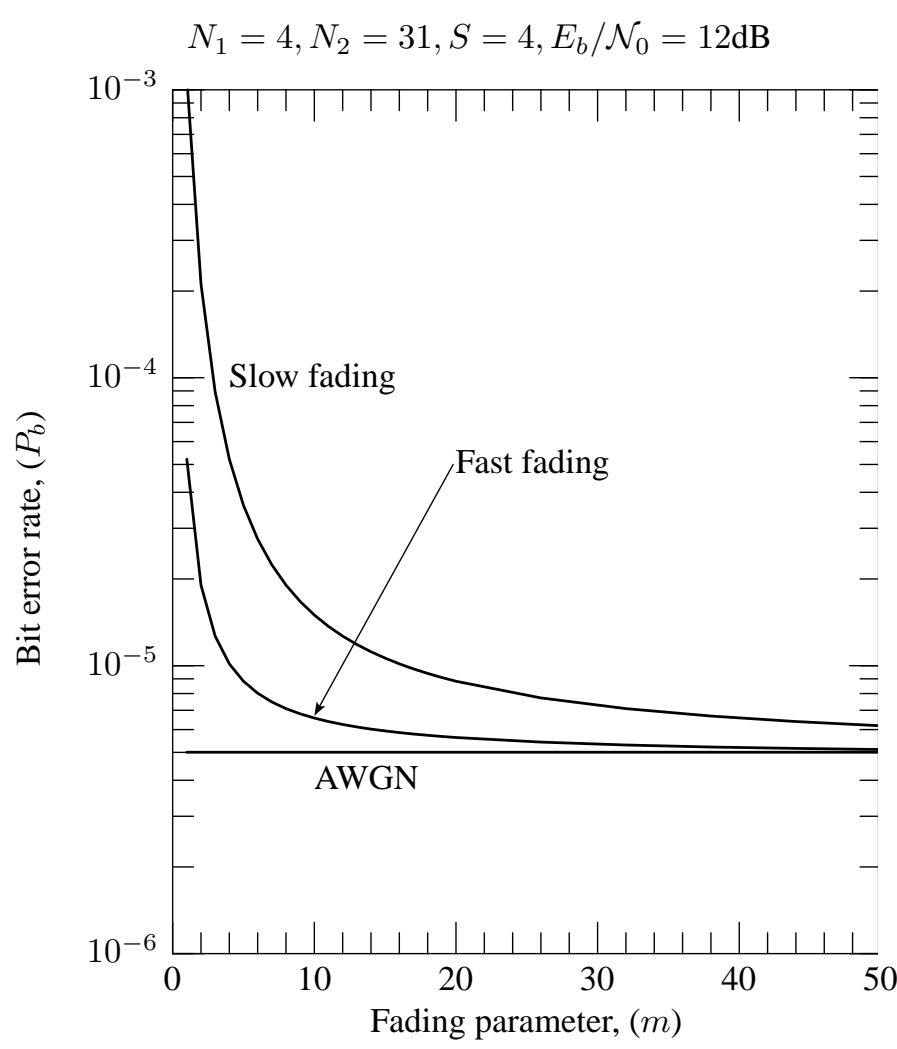

Fig. 5. BER performance versus the Nakagami fading parameter of $m$ for the FS-MC-CDMA systems, when communicating over both the non-fading AWGN, as well as over frequency-selective slow fading and fast fading channels.

ing channel increases. Therefore, the FS MC-CDMA scheme is beneficial for employment over wireless channels exhibiting frequency-selective fading and/or time-selective fading.

\section{REFERENCES}

[1] R. Prasad and S. Hara, "Overview of multicarrier CDMA," IEEE Communications Magazine, pp. 126-133, December 1997.

[2] V. M. Dasilva and E. S. Sousa, "Multicarrier orthogonal CDMA signals for quasi-synchronous communication systems," IEEE Journal on Selected Areas in Communications, vol. 12, pp. 842-852, June 1994.

[3] L. Vandendorpe, "Multitone spread spectrum multiple access communications system in a multipath Rician fading channel," IEEE Transactions on Vehicular Technology, vol. 44, no. 2, pp. 327-337, 1995.

[4] S. Kondo and L. B. Milstein, "Performance of multicarrier DS CDMA systems," IEEE Transactions on Communications, vol. 44, pp. 238-246, February 1996.

[5] E. A. Sourour and M. Nakagawa, "Performance of orthogonal multicarrier CDMA in a multipath fading channel," IEEE Transactions on Communications, vol. 44, pp. 356-367, March 1996.

[6] L.-L. Yang and L. Hanzo, "Performance of generalized multicarrier DSCDMA over Nakagami- $m$ fading channels," IEEE Transactions on Communications, vol. 50, pp. 956 - 966, June 2002.

[7] X. Gui and T. S. Ng, "Performance of asynchronous orthogonal multicarrier CDMA system in frequency selective fading channel," IEEE Transactions on Communications, vol. 47, pp. 1084-1091, July 1999.

[8] M. Progler, C. Evci, and M. Umehira, "Air interface access schemes for broadband mobile systems," IEEE Communications Magazine, pp. 106115, September 1999

[9] J. G. Proakis, Digital Communications. McGraw Hill, 3rd ed., 1995.

[10] A. M. Sayeed and B. Aazhang, "Joint multipath-Doppler diversity in mobile wireless communications," IEEE Transactions on Communications, vol. 47, pp. 123-132, January 1999. 